\title{
Impedance Methods (Apparent Mass, Driving Point Mechanical Impedance and Absorbed Power) for Assessment of the Biomechanical Response of the Seated Person to Whole-body Vibration
}

\author{
Neil J. MANSFIELD
}

Department of Human Sciences, Loughborough University, Loughborough, Leicestershire, LE11 3TU, UK

Received January 21, 2005 and accepted May 2, 2005

\begin{abstract}
Exposure to whole-body vibration is a risk factor for the development of low back pain. In order to develop a fuller understanding of the response of the seated person to vibration, experiments have been conducted in the laboratory investigating the biomechanics of the seated person. Some of these methods are based on the driving force and acceleration at the seat and are reported in the literature as apparent mass, driving point mechanical impedance or absorbed power. This paper introduces the background behind such impedance methods, the theory and application of the methods. It presents example data showing typical responses of the seated human to wholebody vibration in the vertical, fore-and-aft and lateral directions. It also highlights problems that researchers might encounter in performing, analysing and interpreting human impedance data.
\end{abstract}

Key words: Biomechanics, Human response, Mechanical impedance, Apparent mass, Absorbed energy, Absorbed power

\section{Introduction}

Exposure to whole-body vibration is one of several hazards that can result in injury manifesting as low back pain $(\mathrm{LBP})^{1-3)}$. Although a link between vibration and LBP is generally accepted, methods of predicting which individuals are most susceptible to pain remain elusive. One reason that it is difficult to define a good model of risk is that those exposed to vibration are usually also exposed to other hazards, such as heavy lifting, long durations in constrained working postures, regular twisting, and/or cold. Therefore it is very difficult to extract which of these factors caused the pain by using epidemiological methods.

An alternative to the epidemiological approach is to base research methods on a biomechanical approach. With such an approach, raw data can be obtained in the laboratory under controlled conditions using human subjects or animal models. The effects of vibration on the body can then be studied by measuring the mechanical responses of the biological tissue and using these responses to estimate risk of injury. Although this is attractive there are several problems with the biomechanical approach including:

- it is difficult to simulate long term exposures to vibration such as those experienced by workers over their working life;

- ethical constraints mean that it is not usually possible to expose subjects to extreme magnitudes of vibration;

- laboratories required expensive equipment that is safe for human experimentation ('man rated');

- experiments usually involve small populations that might not be representative of those at risk.

Results from biomechanical studies must therefore be used 
Table 1.Categories of whole-body vibration biomechanical research

\begin{tabular}{|c|c|}
\hline Category & Description \\
\hline $\begin{array}{l}\text { Force-motion or } \\
\text { 'impedance' methods }\end{array}$ & $\begin{array}{l}\text { Measurements of driving force and acceleration only made at the 'driving point' } \\
\text { (usually the seat and/or floor) }\end{array}$ \\
\hline $\begin{array}{l}\text { Motion-motion or } \\
\text { 'transmissibility' methods }\end{array}$ & $\begin{array}{l}\text { Comparison of measurements of acceleration at the 'driving point' and measurements } \\
\text { of acceleration made at another location on the body (e.g. head, spine) }\end{array}$ \\
\hline Modelling & $\begin{array}{l}\text { Mathematical, conceptual or physical modelling of the response of the whole body } \\
\text { to vibration, usually based on the results of impedance or transmissibility data (or both) }\end{array}$ \\
\hline Others & $\begin{array}{l}\text { Occasional reports of other types of mechanical response of the body to exposure } \\
\text { to vibration (e.g. bone density, biochemical markers, internal pressure, EMG, etc.) }\end{array}$ \\
\hline
\end{tabular}

with caution, to ensure that inherent limitations in any experimental study are not carried forward into recommendations or guidance. If limitations are taken into account, then biomechanical data can be a powerful tool in augmenting epidemiological evidence to further understanding of vibration risks.

The scope of this paper is to present an overview of one type of biomechanical approach: that of impedance methods. It is designed to explain the types of methods that are found in the literature, the methods for measurement, and to provide some guidance on how to interpret such data. The paper is not designed to provide an exhaustive review of all reports of impedance methods in the literature, but will refer to many of them.

\section{Biomechanical Methods Used in Whole-body Vibration Research}

Most published biomechanical research in the field of whole-body vibration can be broadly categorised into four areas (Table 1). The first two categories involve laboratory (or sometimes field) studies where the dynamics of the whole body are measured. The first category (force-motion or 'impedance' methods) is only concerned with measurements of force and acceleration at the 'driving point' (i.e. the vibrating surface that the subject is in contact with). The second category (motion-motion or 'transmissibility' methods) is only concerned with acceleration measurements made at multiple sites such as measurements at the driving point combined with measurements at the head or spine. The third category of biomechanical research that is commonly found in the literature is that of modelling. Models can take a variety of forms including mathematical, conceptual or physical (e.g. anthropodynamic dummies) and they are often designed to represent impedance or transmissibility data obtained in the first two categories of whole-body vibration biomechanical research ${ }^{1,4)}$. The fidelity of the model depends both on the quality of the model itself but also on the quality of the raw data to which it is designed to represent. The final category of biomechanical research that is found in the literature is that of other methods that have been occasionally reported but have not become commonly applied techniques. Often these are for specific application but some of the methods could be further used in the future. For example, some have reported effects of whole-body vibration on bone density ${ }^{5}$, internal abdominal pressure ${ }^{6,7)}$, EMG response of the erector spinae muscle group 8 , or biochemical markers ${ }^{9}$.

Measurement of the transmission of vibration at sites on the body can be done by mounting accelerometers on the surface of the skin above bony landmarks or other anatomical locations (Fig. 1). In these locations it is important to take the localised movement of the skin into account and to correct for local skin-accelerometer movement artefacts ${ }^{10}$. It is easier to measure vibration at the head as the teeth are rigidly embedded in the skull in the frequency range of interest. Thus, a 'bite-bar' can be placed in the mouths of subjects and used as a mounting point for accelerometers ${ }^{11,12)}$. Measurements on the body can be compared to those at the seat and are usually expressed in terms of transmissibility. Transmissibility $T(f)$ is defined as the ratio of the acceleration at a point on the body $\mathrm{a}_{\text {body }}(f)$ to the acceleration at the seat $\mathrm{a}_{\text {seat }}(f)$ at frequency $f^{3,13)}$ :

$$
T(f)=\frac{\mathrm{a}_{\text {body }}(f)}{\mathrm{a}_{\text {seat }}(f)}
$$

Impedance methods require the measurement of driving force and acceleration at the surface(s) supporting the body. In Fig. 1, a small force plate is shown that can be placed on the surface of a seat to measure the driving force. Measurements of the driving force and acceleration can be used to calculate a range of impedance measures including 


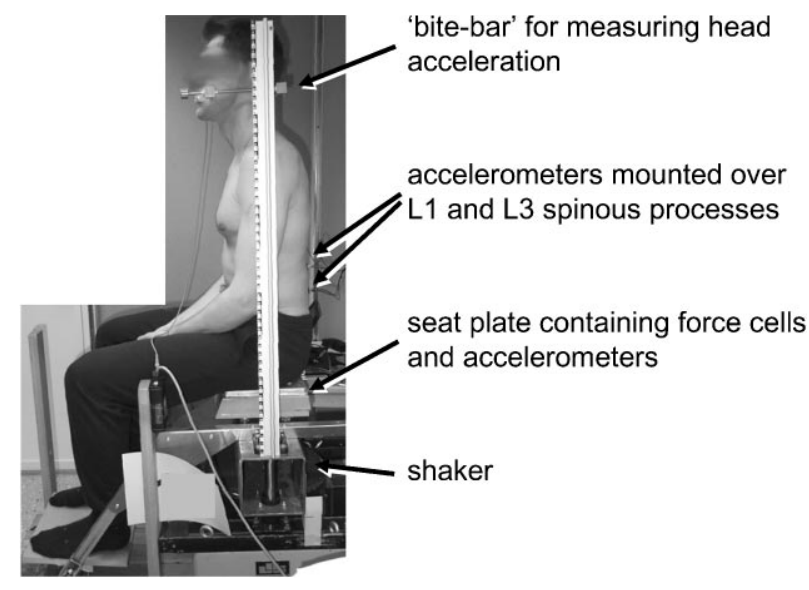

Fig. 1. Typical experimental set-up for measuring transmissibility from the seat to the head or spine and simultaneously measuring the impedance of the body using a force plate.

The laboratory shown in the photograph is situated at the National Institute for Working Life, Umeå, Sweden.

receptance, mobility, accelerance, apparent stiffness, mechanical impedance and apparent mass, although human vibration researchers usually only report the latter two (the others being of most use in other engineering applications). The mechanical impedance or apparent mass can be used to indicate internal resonances in a structure. If this structure is the human body then they might indicate those frequencies of vibration to which the body is most sensitive, mechanically. Impedance methods are also able to provide data that can be used as part of a tool to predict the dynamic response of seats when loaded with a person ${ }^{14)}$. Finally, impedance data can also be used in the design of dummies that can replace human subjects for some types of seat testing ${ }^{15)}$. Therefore, measurements of the mechanical impedance or apparent mass can not only help in furthering understanding of the fundamental responses of the human exposed to vibration but also assist in the design of seats that can be used as protective equipment to provide some isolation from vibration.

Practical force measurement systems usually comprise load cells mounted on a rigid base section and covered with a rigid metal section and are known as 'force plates'. If a force plate is shaken with no additional loading then an output will still be measured due to the inertia of the mass of the rigid metal section. The contribution of this mass must be removed from any measurements to avoid skewing of data.

Most studies investigating the biomechanical response of the human to whole-body vibration have used single axis vibration. Stimuli types have included sinusoidal, random or synthesised vehicle vibration. Irrespective of the stimulus waveform, the biomechanical response of the body is similar, if non-linearities with vibration magnitude are taken into account ${ }^{16)}$.

\section{Mathematical Derivation of Impedance Measures}

The most commonly used impedance measures are the 'driving point mechanical impedance', 'apparent mass' and 'absorbed power'. Although one could argue that absorbed power is not technically an impedance measure, it is classified as such in this paper due to the similarity of its derivation and application to the other 'true' impedance methods. The driving point mechanical impedance is often abridged to 'mechanical impedance' or 'impedance' in whole-body vibration research. In other fields, the 'transfer impedance' is sometimes calculated, where the force and velocity are measured at different locations. There are no known reports of transfer impedance in whole-body vibration research: therefore, 'driving point mechanical impedance' and 'mechanical impedance' can be considered synonymous nouns in this paper.

The driving point mechanical impedance and apparent mass are defined as ratios and calculated as transfer functions. Transfer functions can be calculated using the ratio of the power spectral density (PSD) at the 'input' and the 'output':

$$
T F_{P S D}(f)=\sqrt{\frac{P S D_{\text {output }}(f)}{P S D_{\text {input }}(f)}}
$$

where $T F_{P S D}(f)$ is the transfer function calculated using the PSD method. However, functions are usually calculated using the cross-spectral density (CSD) method defined as ${ }^{1,3)}$ :

$$
T F_{C S D}(f)=\frac{C S D_{\text {input-output }}(f)}{P S D_{\text {input }}(f)}
$$

where $T F_{C S D}(f)$ is the transfer function calculated using the CSD method. The advantage of using the CSD method is that the function generates the phase of the response and also only includes data at the input and output that are correlated, thus reducing the effects of noise in the measurement system. The extent of the correlation between the input and output can be expressed in terms of the coherence:

$$
\text { coherence }(f)^{2}=\frac{\left|C S D_{\text {input-output }}(f)\right|^{2}}{P S D_{\text {input }}(f) \times P S D_{\text {output }}(f)}
$$

Coherence is a value between 0 and 1: the greater the coherence the greater the correlation between the two signals being analysed. 
A full report of any measure of a transfer function such as the apparent mass, mechanical impedance or transmissibility should include the modulus, phase and coherence. The coherence indicates the confidence that one can have with data and should be considered when interpreting the data. The phase is important when modelling and considering relative motion between two points. However, the modulus is usually considered the most important part of any impedance measurement as it indicates those frequencies where the body is most responsive to the driving vibration.

\section{Driving point mechanical impedance}

The driving point mechanical impedance, $Z(f)$, is defined as the complex ratio of the force to the velocity at frequency, $f$ :

$$
Z(f)=\frac{F(f)}{v(f)}
$$

where $F(f)$ is the force and $v(f)$ is the velocity at frequency $f$. The units of impedance are $\mathrm{Ns} / \mathrm{m}$. Usually the velocity is not measured directly but calculated from acceleration which can be done either in the time domain by integrating the acceleration time history, or in the frequency domain by using:

$$
a(f)=2 \pi f \times v(f)
$$

where $a(f)$ is the acceleration at frequency $\mathrm{f}$, such that:

$$
Z(f)=2 \pi f \times \frac{F(f)}{a(f)}
$$

The mechanical impedance of a rigid mass is a function of frequency. The greater the mass, the greater the gradient (Fig. 2). For human vibration research, this means that it is difficult to compare results for different subjects if they have different weights. The modulus of the mechanical impedance should have an origin at zero and the phase should have an origin at $90^{\circ}$.

\section{Normalised mechanical impedance}

As the gradient of the mechanical impedance is a function of the weight of the object being measured, it is sometimes desirable to normalise data such that results from subjects of different weights can be directly compared. One method of normalisation is to select the lowest frequency where data show high coherence, and to divide the mechanical impedance at all frequencies by the data at the selected frequency, resulting in a dimensionless quantity. For example, Holmlund et al. ${ }^{17)}$ normalise data at $2 \mathrm{~Hz}$, such that all mechanical impedance data equal 1.0 at $2 \mathrm{~Hz}$.
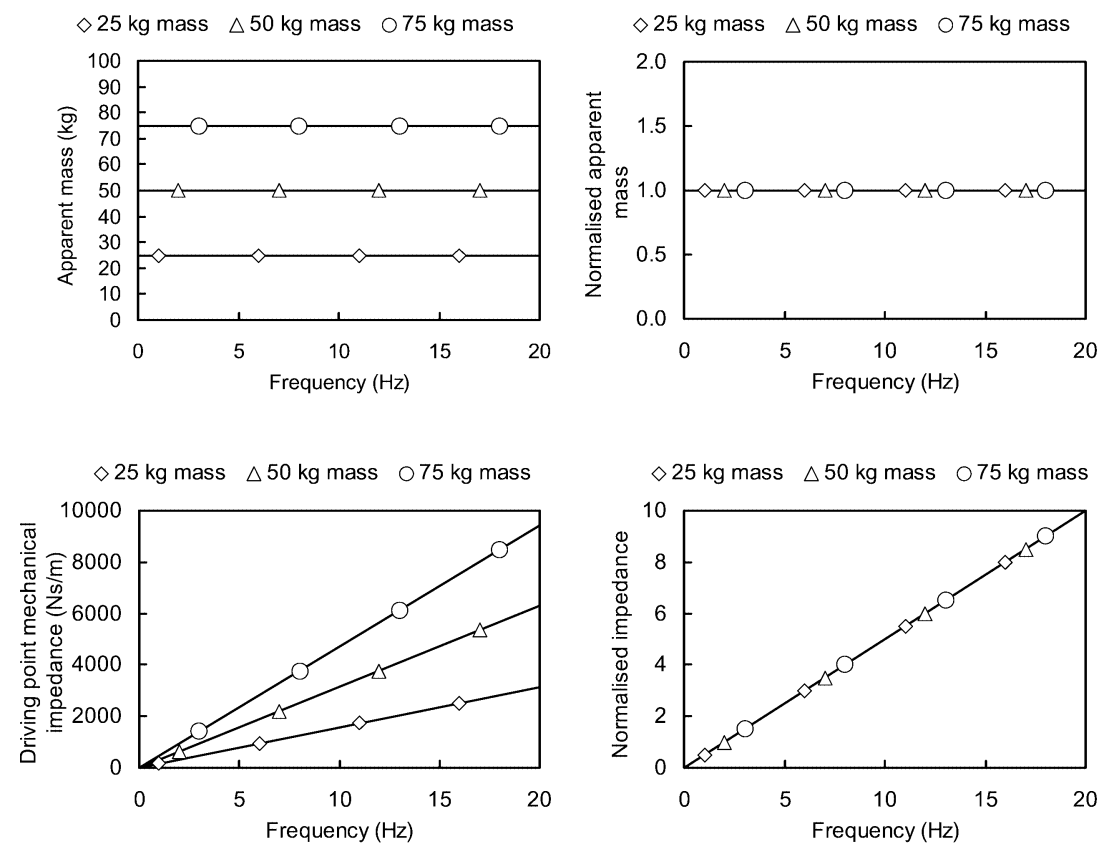

Fig. 2. Apparent mass, normalised apparent mass, driving point mechanical impedance and normalised mechanical impedance for rigid masses of 25,50 and $75 \mathrm{kgs}$. Normalised impedance is normalised at $2 \mathrm{~Hz}$. 
For a rigid mass, the normalised mechanical impedance is a straight line with a gradient that depends on the frequency of normalisation (Fig. 2).

\section{Apparent mass}

Netwon's second law states that "the rate of change of momentum of a body is proportional to the force acting on it and is in the direction of the force" and can be summarised by the equation:

$$
F=m a
$$

where $m$ is the mass. For a rigid mass, this equation holds at all frequencies. However, for a non-rigid system, such as the human body, the force required to accelerate the supporting surface is a complex function of frequency. This function is presented in terms of the 'apparent mass' which is defined as:

$$
M(f)=\frac{F(f)}{a(f)}
$$

where $M(f)$ is the apparent mass at frequency $f$. The apparent mass is usually calculated in the frequency domain using the CSD method. The units of apparent mass are kg.

The apparent mass of a rigid mass is not a function of frequency but is equal to its static mass (Fig. 2). For human subjects, this means that the apparent mass is not only a function of their dynamic characteristics but also of their supported weight (i.e. the weight of the person supported on the force plate). This can make it difficult to compare data between subjects of different weights. The apparent mass modulus should tend towards an origin that is equal to the supported weight and the phase should have an origin of $0^{\circ}$.

\section{Normalised apparent mass}

As the apparent mass is a function of the supported weight, it is often desirable to normalise data such that results from subjects of different weights can be directly compared. Normalisation is usually carried out by dividing the modulus of the apparent mass by the supported weight, resulting in a dimensionless quantity:

$$
M_{\text {normalised }}(f)=\frac{M(f)}{m}
$$

where $m$ is the supported weight and $M_{\text {normalised }}(f)$ is the normalised apparent mass at frequency $f$. If the entire body weight is supported on the force plate (e.g. for standing subjects or those sitting with legs hanging free) then the supported weight is the same as the body weight. If feet are supported then the supported weight is often assumed to equal the apparent mass at the lowest frequency where data show high coherence.

The normalised apparent mass of a rigid mass has a value of 1.0 at all frequencies, irrespective of the weight (Fig. 2).

\section{Absorbed power}

Power is classically defined as the product of the force and the velocity in the time domain:

$$
P(t)=F(t) v(t)
$$

As both quantities are vectors, they have direction and therefore $P$ is complex ${ }^{3,18,19)}$. The real part of the complex power reflects the energy absorbed by the structure whereas the imaginary part reflects the energy that is cyclically stored and dissipated back to the driving point by elastic components in the system. Elastic power averages to zero over extended periods of time whereas the absorbed power accrues with exposure $^{20)}$. The absorbed power can be defined as:

$$
P_{a b s}(f)=/ G_{f v}(f) \mid \cos \phi_{f v}(f)
$$

where $\left|G_{f v}(f)\right|$ is the modulus and $\phi_{f v}(f)$ is the phase of the cross-spectrum between the force and the velocity. When the force and velocity are in phase, all of the energy is in the real part and is absorbed; when the force and velocity are $90^{\circ}$ out of phase all of the energy is in the imaginary part and is not absorbed. The units of absorbed power are $\mathrm{Nms}^{-1}$ or $\mathrm{W}$.

For rigid masses, the force and velocity are phase shifted by $90^{\circ}$ and therefore there is no net absorption of energy. Thus, absorbed power is zero for rigid masses. For compliant systems, such as the human body, the absorbed power is a function of the vibration magnitude and also the mass of the subject. As the absorbed power is a function of vibration magnitude, it makes the quantity sensitive to vibration spectral content. Therefore, any fluctuations in the spectrum of acceleration used on a shaker system will be reflected in the measurements of the absorbed power. Furthermore, the absorbed power will shift in magnitude as the magnitude of the vibration increases. These two properties of absorbed power are in contrast to the properties of mechanical impedance and apparent mass which are both ratio measures and therefore are not directly proportional to stimulus magnitude. The shift in the magnitude of the absorbed power with body weight is similar to that observed for apparent mass and mechanical impedance.

\section{Normalised absorbed power}

To enable comparison of the absorbed power between 
subjects of different weights, it is often desirable to normalise by division by the static sitting weight, to provide a measurement in $\mathrm{W} / \mathrm{kg}$. The sitting weight depends on posture (e.g. legs free hanging or resting on a footrest) and can be determined by calculating the apparent mass and defining the sitting weight as the apparent mass modulus at a low frequency (e.g. $1 \mathrm{~Hz}$ ). In addition to normalisation by sitting weight, it is also often desirable to remove effects of vibration spectrum on the absorbed power by dividing the absorbed power by the power spectrum of the acceleration to provide data with units of $\mathrm{Ns}^{3} \mathrm{~m}^{-1} / \mathrm{kg}$.

\section{Conversion Between Results Reported Using Alternative Impedance Methods}

It is possible to convert data between mechanical impedance and apparent mass using the transformation:

$$
Z(f)=2 \pi f \times M(f)
$$

for the modulus. The phase is converted by applying a $90^{\circ}$ shift.

If data have been normalised, it is not possible to recalculate the non-normalised results, unless the supported weights are known. However, it is possible to convert data between normalised metrics by performing the transformation in equation (13) and then re-normalising the transformed data.

Although the absorbed power is calculated from the same raw data as the apparent mass and mechanical impedance, it is not possible to directly transform data between the quantities. It is only possible to perform the transformation from impedance methods to absorbed power if the modulus and phase of the impedance method is known, in addition to the specific spectrum of the vibration used for the experiment. It is not possible to convert from absorbed power to impedance measures as phase data is lost during the calculation.

\section{Comparison of Results Obtained Using Alternative Methods of Analysis of Biomechanical Data}

One of the classic models of the biomechanical response of the seated person is that of Fairley and Griffin ${ }^{21}$. This model was developed from a large study where the apparent masses of 60 people were measured. The average apparent mass from this cohort was used to specify parameters for a two mass single degree-of-freedom model (moving mass: $45.6 \mathrm{~kg}$; base mass: $6.0 \mathrm{~kg}$; damping ratio: 0.475 ; damping: 1,360 Ns/m; natural frequency: $5.0 \mathrm{~Hz}$ ). The dynamic response of this model has been used to provide source data to enable a comparison of results that would be obtained using the alternative impedance methods considered in this paper. For measurements of mechanical impedance, the model has a modulus response characterised by increasing with frequency up to a peak at $5.50 \mathrm{~Hz}$ (Fig. 3). At low frequencies, the increase is proportional to frequency, similar
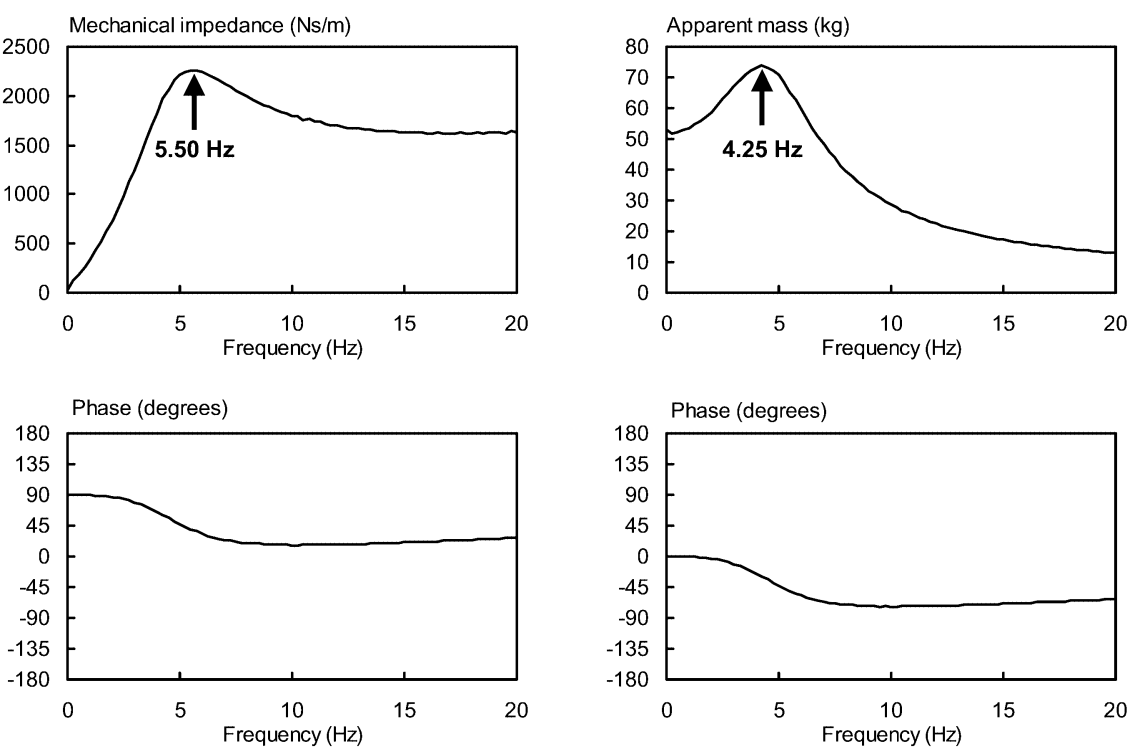

Fig. 3. Mechanical impedance and apparent mass of a two mass single degree-of-freedom model of the dynamic response of the seated human exposed to vibration in the frequency range 0 to $20 \mathrm{~Hz}$. 

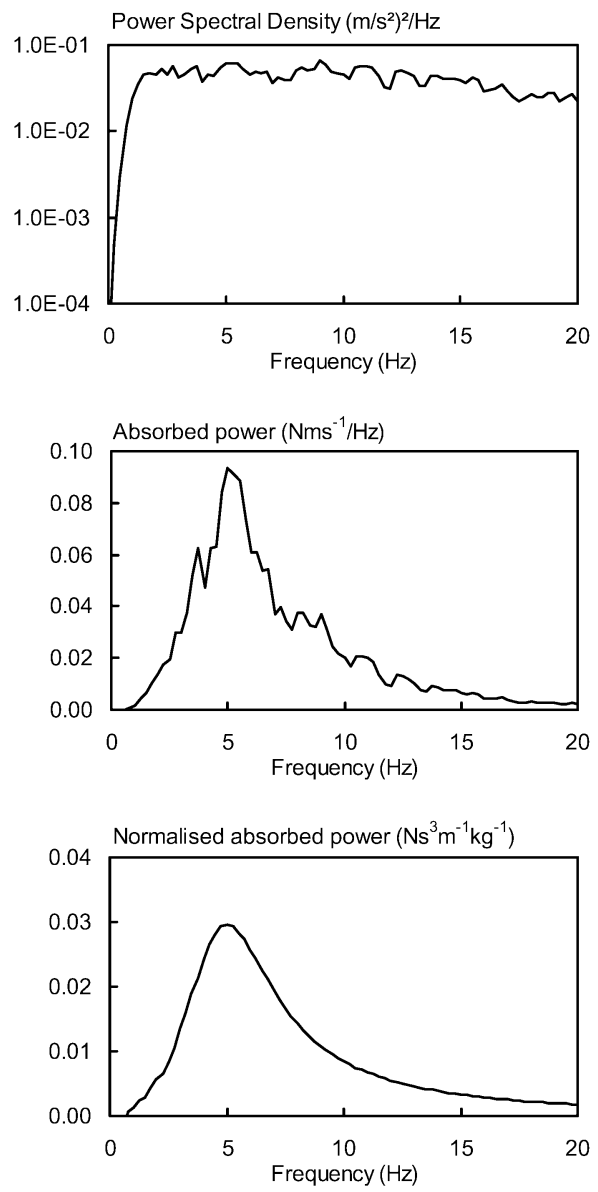

Fig. 4. Power spectral density of random vibration in the frequency range of 1 to $20 \mathrm{~Hz}$ and with a magnitude of $1.0 \mathrm{~m} / \mathrm{s}^{2}$ r.m.s. and corresponding absorbed power and normalised absorbed power for a two mass single degree-of-freedom model of the dynamic response of the seated human.

to that which occurs for a rigid mass of $51.6 \mathrm{~kg}$. The response decreases at frequencies above the peak. The phase of the mechanical impedance has an origin at $90^{\circ}$. The phase lead decreases as the frequency increases up to about $5.50 \mathrm{~Hz}$.

The apparent mass modulus has an origin which corresponds to the weight of the system $(51.6 \mathrm{~kg})$. The apparent mass has a peak at $4.25 \mathrm{~Hz}$ with a magnitude of 74 $\mathrm{kg}$. At higher frequencies the apparent mass decreases with frequency. The phase of the apparent mass has an origin at zero and has an identical shape to that for the mechanical impedance.

The most important difference between the measurements of impedance made using the two different methods is that

\footnotetext{
* Differences between the peak frequencies measured using mechanical impedance and apparent mass are most apparent for highly damped systems such as the human body, and might not be important for other types of structure.
}

the frequency of the peak is different for the same system being measured*. The frequency of the peak in the mechanical impedance is always either the same or higher to that observed for the apparent mass. Therefore, frequencies of the peaks in mechanical impedance and apparent mass cannot be directly compared. For a comparison to be made, the transfer functions must be transformed and the new resonance frequency extracted. For simple single degreeof-freedom apparent mass models (such as the one considered here), the resonance in the base-to-moving mass transmissibility occurs at the same frequency as the peak in the apparent mass. This illustrates how the apparent mass gives a more direct indication of the biomechanical response of the structure and is therefore the preferred method of presentation for most researchers.

The absorbed power for the Fairley and Griffin model is shown in Fig. 4. This has a peak at about $5 \mathrm{~Hz}$. To simulate laboratory techniques as closely as possible, the response of the model was calculated in the time domain. As it is not possible to test using infinite data series, the power spectral density of the 'stimulus' acceleration was not perfectly flat. As the absorbed power is a function of the acceleration, the quantity does not generate a smooth curve, even for a simple mathematical model being tested using a computer simulation. Features in the power spectrum are transferred to the absorbed power (e.g. in Fig. 4, features between 10 and $15 \mathrm{~Hz}$ in the PSD of the acceleration can be clearly observed in the absorbed power). Normalising the absorbed power by the acceleration spectrum smoothes the curve. It should be noted that this type of normalisation and 'smoothing' is numerically exact and does not result in loss of data or precision as can occur by other forms of smoothing that are often applied in other fields of biomechanics.

\section{Example Impedance Data from the Literature}

There is a growing body of literature reporting the impedance of the human body. For whole-body vibration, important contributions have been made by many, including: Fairley ${ }^{21,22)}$, Holmlund ${ }^{18,23-26)}$, Mansfield ${ }^{16,19,27-32)}$, Matsumoto ${ }^{33,34)}$, Miwa ${ }^{35)}$, Nawayseh $^{36,37)}$, Rakheja ${ }^{38-42)}$, and Smith ${ }^{43,44)}$. Although contributions have been made by many individuals, example data presented here will be sourced primarily from the author's own work due to availability of raw data and knowledge of all experimental conditions.

Typical apparent mass data of seated subjects exposed to vertical whole-body vibration are shown in Fig. 5. Subjects were exposed to vertical random vibration with a magnitude of $1.0 \mathrm{~m} / \mathrm{s}^{2}$ r.m.s. and no backrest was used in the experiment. 

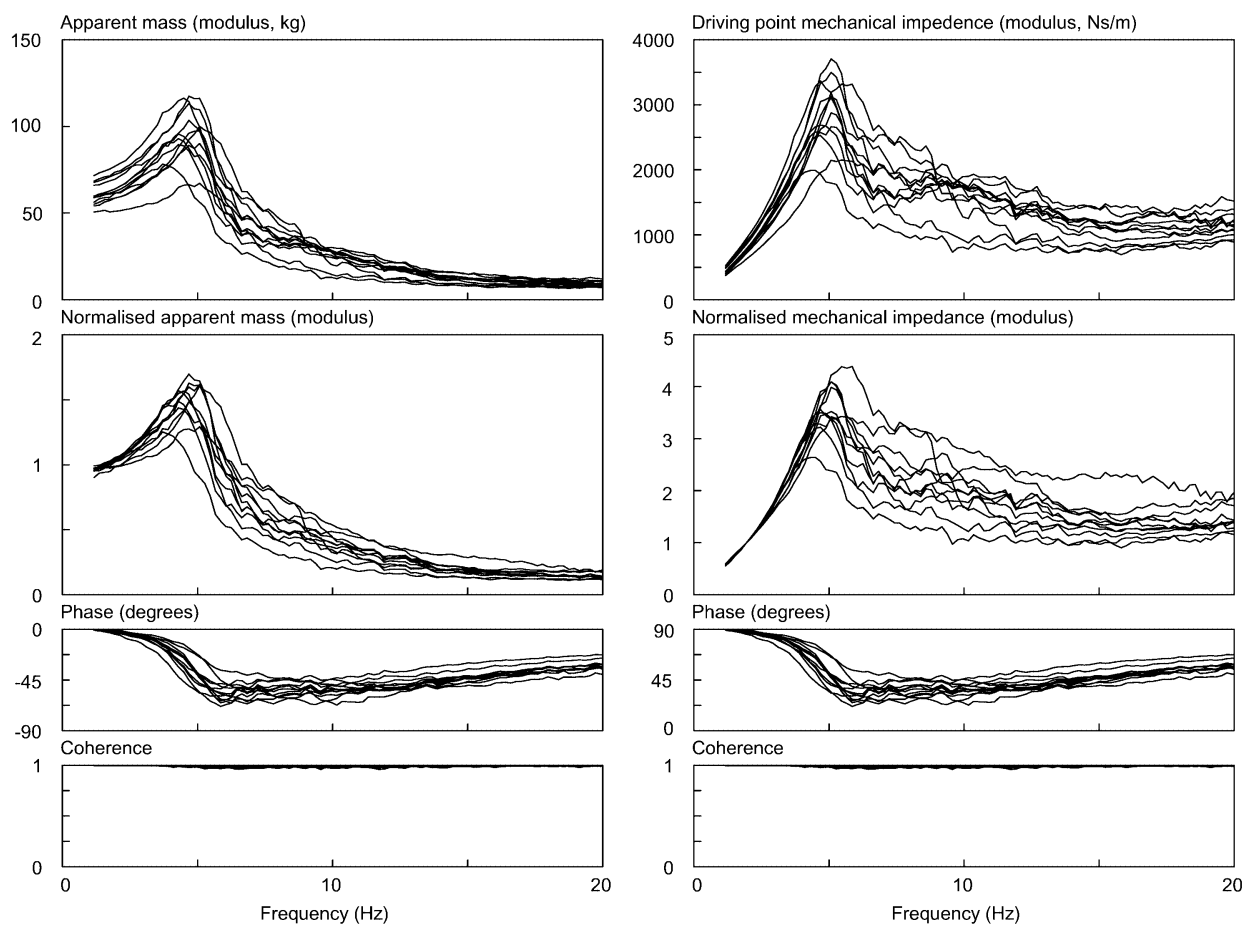

Fig. 5. Apparent mass, normalised apparent mass, mechanical impedance and normalised mechanical impedance (normalised to $2 \mathrm{~Hz}$ ) modulus, phase and coherence of the biomechanical response of 12 seated subjects exposed to random vertical vibration in the frequency range of 1 to $20 \mathrm{~Hz}$ and with a magnitude of $1.0 \mathrm{~m} / \mathrm{s}^{2}$ r.m.s. Data from Mansfield and Griffin, $2002^{27}$.

It can be seen that the apparent masses of most subjects have a peak at just below $5 \mathrm{~Hz}$ but the mechanical impedance has a peak at just above $5 \mathrm{~Hz}$. At frequencies below resonance the effect of subject weight on the apparent mass can be observed. This scaling of data by subject weight is removed if the data are normalised.

The peak in the apparent mass and mechanical impedance is non-linear with vibration magnitude. At low magnitudes of vibration the peak occurs at a higher frequency than at high magnitudes (Fig. 6). This phenomenon is known as a 'softening effect'. The softening effect can also be observed in the absorbed power ${ }^{28,32)}$ (Fig. 7).

In the horizontal directions, the apparent mass depends on the backrest condition. If there is a backrest, the fore-aft peak occurs at about $4 \mathrm{~Hz}$; without a backrest it occurs at about $1 \mathrm{~Hz}$, with a second peak at approximately $2 \mathrm{~Hz}$. In the lateral direction, the peak occurs at about $2 \mathrm{~Hz}$ irrespective of backrest condition ${ }^{22,30)}$ (Fig. 8).

Although much of the data reported in the literature has been obtained for adult subjects sitting in an upright posture, there is a growing body of work that has investigated the biomechanical response of the seated subject in semi-reclined postures and with a steering whee ${ }^{38,40)}$. Although data

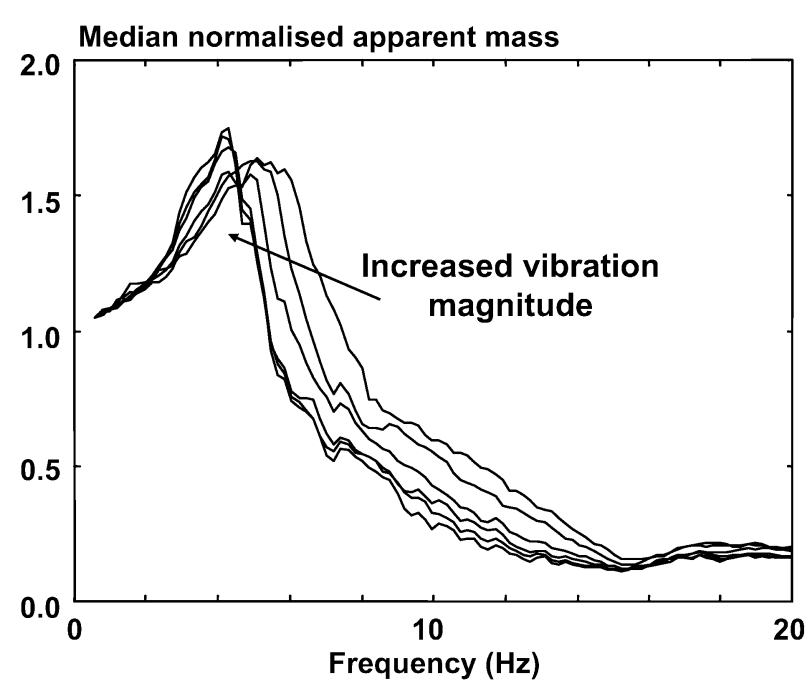

Fig. 6. Median normalised apparent masses of 12 subjects exposed to vertical vibration at $0.25,0.5,1.0,1.5,2.0$ and $2.5 \mathrm{~m} / \mathrm{s}^{2}$ r.m.s. Resonance frequencies decrease with increasing vibration magnitude. Adapted from Mansfield and Griffin, 200029).

obtained in all postures have a broadly similar shape, there are important differences. For example, Rakheja et al. (2002) showed that the magnitude of the peak in apparent mass 


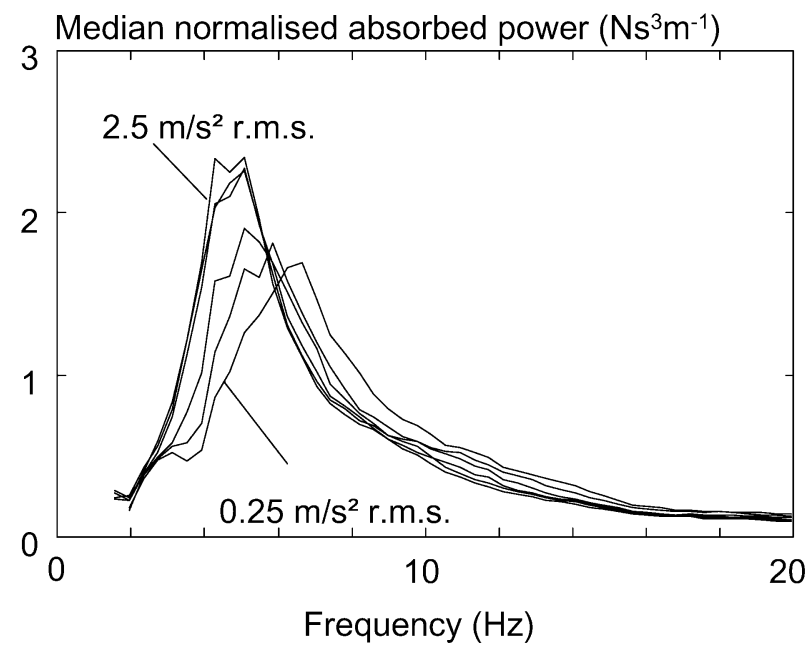

Fig. 7. Median normalised absorbed power for 12 subjects exposed to vertical vibration at $0.25,0.5,1.0,1.5,2.0$ and $2.5 \mathrm{~m} / \mathrm{s}^{2}$ r.m.s. Resonance frequencies decrease with increasing vibration magnitude. Adapted from Mansfield and Griffin, 1998 ${ }^{19}$. (NB normalisation with respect to vibration magnitude only). reduces with the use of a steering wheel and that the semireclined posture causes the peak in apparent mass to increase. There have been some studies of the biomechanical response of children which have shown that, even for children weighting less than $18 \mathrm{~kg}$, the general response is similar to that of adults, albeit with several differences ${ }^{45}$.

\section{Specific Areas for Caution in Interpretation of Experiment Results}

Care must be taken when interpreting biomechanical data reported in the literature. The most common problem is one of the effects of averaging, where there is no easy solution. Consider the responses of five subjects where each subject has a different resonance frequency in the apparent mass (Fig. 9). In this example, the peak normalised apparent mass is 1.45 for each subject. If the mean of the data is calculated then a smooth curve is generated, but the magnitude of the peak is lower than the magnitude of the peak observed for
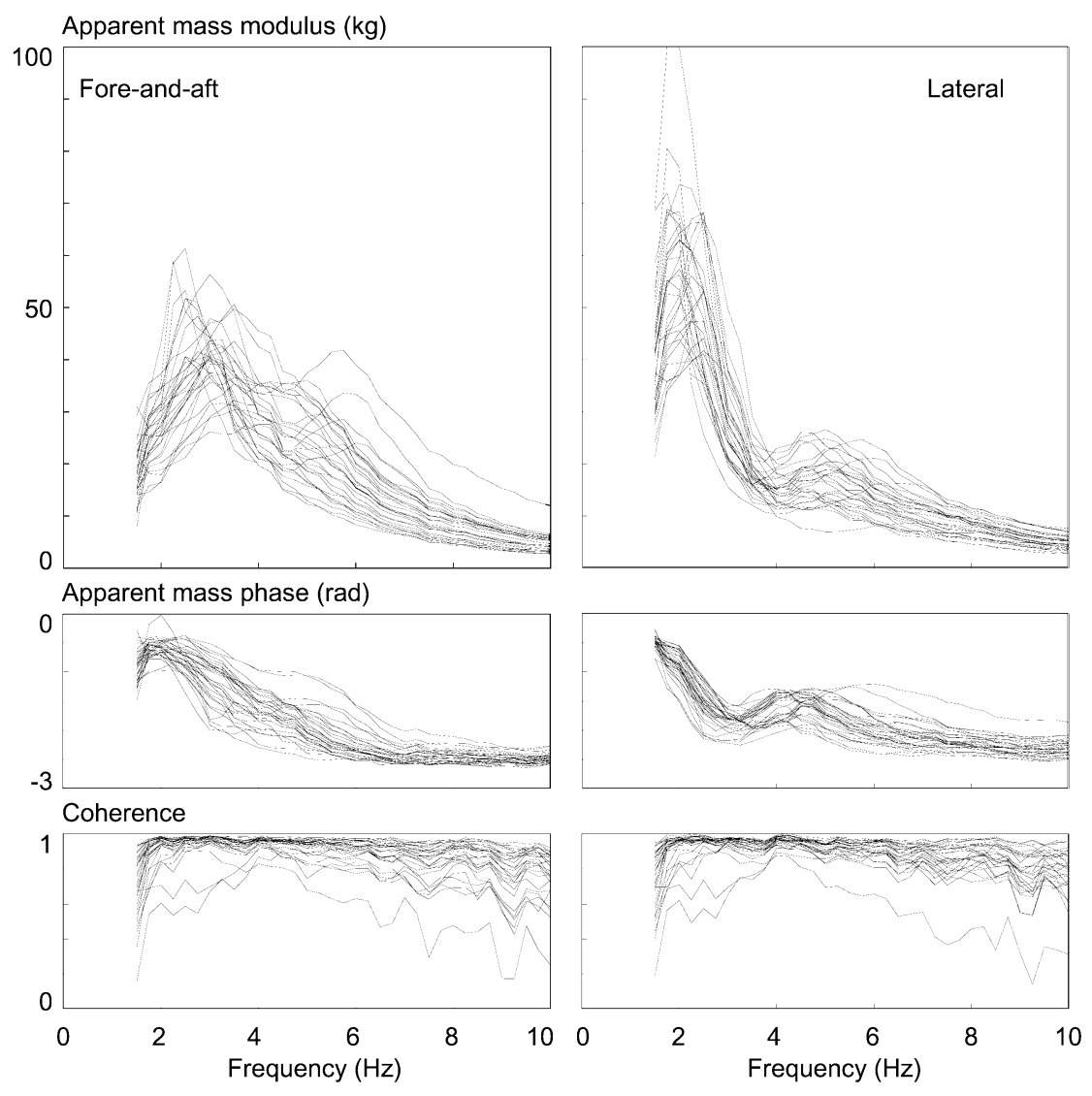

Fig. 8. Apparent masses of 30 subjects measured using horizontal vibration in the fore-andaft and lateral directions (no backrest).

Data from Mansfield and Lundström, 1999 ${ }^{30}$. (NB the peak that occurs at about $1 \mathrm{~Hz}$ in the fore-aft direction is not shown in this figure due to constrains in the experimental set-up). 

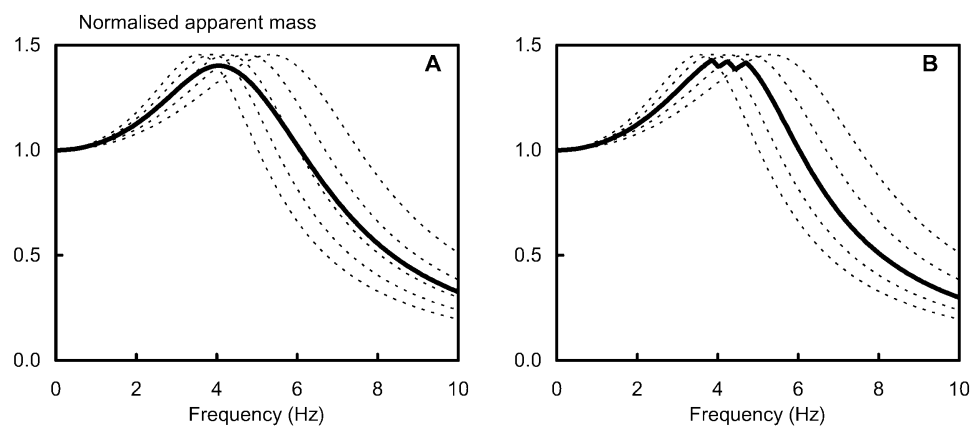

Fig. 9. Five normalised apparent masses (- . - - ) with different resonance frequencies and (A) mean normalised apparent mass and (B) median normalised apparent mass.

any of the five individual subjects. Therefore, the mean data implies a more highly damped system than occurs for any subject. A similar effect occurs if the median apparent mass is calculated, although in this case the response is not smooth as it follows the response curves of different subjects. One partial solution to this problem is to ensure that when the mean apparent mass resonance frequency is reported, this is based on the mean of individual subjects' resonance frequencies, rather than the frequency of the peak from the mean transfer function.

Most reports of the apparent masses of subjects have predominantly used relatively young adults as subjects. Although there are many individual data series in the literature that correspond to older subjects, it is possible that any age effect that might occur is masked by the dominance of younger experimental participants.

Care is required in the interpretation of resonance frequency data that can be reported to a misleading degree of precision. If experiments are completed using sample rates that are a power of two (e.g. 512 or 1,024 samples/s) then spectral analysis can be completed with convenient frequency increments that are either integers or simple fractions. For example, frequency domain data could be reported where each point occurs at 0.25 or $0.5 \mathrm{~Hz}$ increments. If other sample rates are used then frequency domain data cannot be reported using such increments. For example, if a sample rate of 200 samples/s is used, frequency domain data is likely to be reported with a resolution of $0.391 \mathrm{~Hz}$. Although the results are valid, reports of individual subject data could be interpreted as having a greater precision than is the genuine case. Reports of individual subject data should always specify the frequency resolution. The problem is avoided if sample rates are selected to be at or close to a power of two.

Almost all biomechanical data in the literature has been collected using single-axis vibration and using stationary random signals. Although this is attractive in terms of keeping to simple stimuli, it is not representative of workplaces, where vibration is usually complex, non-stationary and multi-axis. Although much has been learned using such simple experimental approaches, major future developments in the field will require experiments using multi-axis complex stimuli.

\section{References}

1) Mansfield NJ (2004) Human Response to Vibration. CRC Press, Boca Raton, Florida, USA.

2) European Commission (2002) Directive 2002/44/EC of the European Parliament and of the Council of 25 June 2002 on the minimum health and safety requirements regarding the exposure of workers to the risks arising from physical agents (vibration) (sixteenth individual Directive within the meaning of Article 16(1) of Directive 89/391/EEC). Official Journal of the European Communities L177, 13-9.

3) Griffin MJ (1990) Handbook of human vibration. Academic, London.

4) Griffin MJ (2001) The validation of biodynamic models. Clin Biomech 16 (Suppl 1), S81-92.

5) Rubin C, Recker R, Cullen D, Ryaby J, McCabe J, McLeod K (2004) Prevention of postmenopausal bone loss by a low-magnitude, high-frequency mechanical stimuli: A clinical trial assessing compliance, efficacy, and safety. J Bone Miner Res 19, 343-51.

6) Village J, Morrison J, Robinson D (1993) Internal pressure response to mechanical shocks of varying frequency and amplitude. In: The United Kingdom Group Meeting on Human Response to Vibration, held at the Army Personnel Research Establishment. 
7) Robinson DG, Morrison JB, Cameron BJ (1995) The contribution of muscle response and internal pressure to estimation of spinal compression from mechanical shocks using a simple biomechanical model. In: The United Kingdom Group Meeting on Human Response to Vibration, held at Silsoe research institute.

8) Zimmermann CL, Cook TM, Goel VK (1993) Effects of seated posture on erector spinae EMG activity during whole body vibration. Ergonomics 36, 667-75.

9) Bosco C, Iacovelli $M$, Tsarpela $O$, Cardinale $M$, Bonifazi M, Tihanyi J, Viru M, De Lorenzo A, Viru A (2000) Hormonal responses to whole-body vibration in men. Eur J Appl Physiol 81, 449-54.

10) Kitazaki S, Griffin MJ (1995) A data correction method for surface measurement of vibration on the human body. J Biomech 28, 885-90.

11) Paddan GS, Griffin MJ (1988) The transmission of translational seat vibration to the head-I. Vertical seat vibration. J Biomech 21, 191-8.

12) Paddan GS, Griffin MJ (1988) The transmission of translational seat vibration to the head-II. Horizontal seat vibration. J Biomech 21, 199-206.

13) International Organization for Standardization (1990) Glossary of terms relating to mechanical vibration and shock. ISO 2041.

14) Wei L, Griffin MJ (1998) The prediction of seat transmissibility from measures of seat impedance. $\mathrm{J}$ Sound Vib 214, 121-37.

15) Lewis CH, Griffin MJ (2002) Evaluating the vibration isolation of soft seat cushions using an active anthropodynamic dummy. J Sound Vib 253, 295-311.

16) Mansfield NJ, Maeda S (2005) Comparison of the apparent mass of the seated human measured using random and sinusoidal vibration. Ind Health 43, 233 40.

17) Holmlund P, Lundström R, Lindberg L (2000) Mechanical impedance of the human body in the vertical direction. Appl Ergonomics 31, 415-22.

18) Lundström R, Holmlund P, Lindberg L (1998) Absorption of energy during vertical whole-body vibration exposure. J Biomech 31, 317-26.

19) Mansfield NJ, Griffin MJ (1998) Effect of magnitude of vertical whole-body vibration on absorbed power for the seated human body. J Sound Vib 215, 813-26.

20) Holmlund $P$ (1998) Absorbed power and mechanical impedance of the seated human exposed to whole-body vibration in horizontal and vertical directions. $\mathrm{PhD}$ Thesis, Umeå University, Sweden.

21) Fairley TE, Griffin MJ (1989) The apparent mass of the seated human body: vertical vibration. J Biomech 22, 81-94.

22) Fairley TE, Griffin MJ (1990) The apparent mass of the seated human body in the fore-and-aft and lateral directions. J Sound Vib 139, 299-306.

23) Holmlund P, Lundström R (2001) Mechanical impedance of the sitting human body in single-axis compared to multi-axis whole-body vibration exposure. Clin Biomech 16 (Suppl 1), S101-10.

24) Holmlund $P$ (1999) Absorbed power and mechanical impedance of the seated human measured within a real vehicle environment compared with single axis laboratory data. J Low Freq Noise Vib Active Control 18, 97-110.

25) Holmlund P, Lundström R (1998) Mechanical impedance of the human body in the horizontal direction. J Sound Vib 215, 801-12.

26) Lundström R, Holmlund $P$ (1998) Absorption of energy during whole-body vibration exposure. J Sound Vib 215, 789-800.

27) Mansfield NJ, Griffin MJ (2002) Effects of posture and vibration magnitude on apparent mass and pelvis rotation during exposure to whole-body vertical vibration. J Sound Vib 253, 93-107.

28) Mansfield NJ, Holmlund P, Lundström R (2001) Apparent mass and absorbed power during exposure to whole-body vibration and repeated shocks. J Sound Vib 248, 427-40.

29) Mansfield NJ, Griffin MJ (2000) Non-linearities in apparent mass and transmissibility during exposure to whole-body vertical vibration. J Biomech 33, 933-41.

30) Mansfield NJ, Lundström R (1999) The apparent mass of the human body exposed to non-orthogonal horizontal vibration. J Biomech 32, 1269-78.

31) Mansfield NJ, Lundström R (1999) Models of the apparent mass of the seated human body exposed to horizontal whole-body vibration. Aviat Space Environ Med 70, 1166-72.

32) Mansfield NJ, Holmlund P, Lundström R (2000) Comparison of subjective responses to vibration and shock with standard analysis methods and absorbed power. J Sound Vib 230, 477-91.

33) Matsumoto Y, Griffin MJ (2002) Effect of muscle tension on non-linearities in the apparent masses of seated subjects exposed to vertical whole-body vibration. J Sound Vib 253, 77-92.

34) Matsumoto $Y$, Griffin MJ (2001) Modelling the dynamic mechanisms associated with the principal resonance of the seated human body. Clin Biomech (Bristol, Avon) 
16(Suppl 1), S31-44.

35) Miwa $T$ (1973) Mechanical impedance of the human body in various postures. Ind Health 13, 1-22.

36) Nawayseh N, Griffin MJ (2003) Non-linear dual-axis biodynamic response to vertical whole-body vibration. J Sound Vibrat 268, 503-23.

37) Nawayseh N, Griffin MJ (2004) Tri-axial forces at the seat and backrest during whole-body vertical vibration. J Sound Vibrat 277, 309-26.

38) Wang W, Rakheja S, Boileau PE (2004) Effects of sitting postures on biodynamic response of seated occupants under vertical vibration. Int J Ind Ergonomics 34, 289306.

39) Boileau PE, Rakheja S, Wu X (2002) A body mass dependent mechanical impedance model for applications in vibration seat testing. J Sound Vib 253, 243-64.

40) Rakheja S, Haru I, Boileau PE (2002) Seated occupant apparent mass characteristics under automotive postures and vertical vibration. J Sound Vib 253, 57-75.

41) Wu X, Rakheja S, Boileau PÉ (1999) Analyses of relationships between biodynamic response functions. J Sound Vib 226, 595-606.

42) Boileau PÉ, Wu X, Rakheja S (1998) Definition of a range of idealized values to characterize seated body biodynamic response under vertical vibration. J Sound Vib 215, 841-62.

43) Smith SD (2000) Modeling differences in the vibration response characteristics of the human body. J Biomech 33, 1513-16.

44) Smith SD, Kazarian LE (1994) The effects of acceleration on the mechanical impedance response of a primate model exposed to sinusoidal vibration. Ann Biomed Eng 22, 78-87.

45) Giacomin J (2004)Apparent mass of small children: experimental measurements. Ergonomics 47, 1454-74. 